\title{
ERRATUM TO THE PAPER "EXTINCTION AND ERGODIC STATIONARY DISTRIBUTION OF A MARKOVIAN-SWITCHING PREY-PREDATOR MODEL WITH ADDITIONAL FOOD FOR PREDATOR"
}

\author{
Xiaoxia Guo ${ }^{1,2}$ And Dehao Ruan ${ }^{3,4, *}$
}

\begin{abstract}
In this work we have studied a stochastic predator-prey model where the prey grows logistically in the absence of predator. All parameters but carrying capacity have been perturbed with telephone noise. The prey's growth rate and the predator's death rate have also been perturbed with white noises. Both of these noises have been proved extremely useful to model rapidly fluctuating phenomena Dimentberg (1988). The conditions under which extinction of predator and prey populations occur have been established. We also give sufficient conditions for positive recurrence and the existence of an ergodic stationary distribution of the positive solution, red which in stochastic predator-prey systems means that the predator and prey populations can be persistent, that is to say, the predator and prey populations can be sustain a quantity that is neither too much nor too little. In our analysis, it is found that the environmental noise plays an important role in extinction as well as coexistence of prey and predator populations. It is shown in numerical simulation that larger white noise intensity will lead to the extinction of the population, while telephone noise may delay or reduce the risk of species extinction.
\end{abstract}

Mathematics Subject Classification. 60G28, 34A12, 34K34.

Received October 5, 2020. Accepted September 30, 2020.

Math. Model. Nat. Phenom. 15 (2020) 46, https://doi.org/10.1051/mmnp/2019055

\section{Corrections}

(1) The name of the corresponding author should be Dehao Ruan instead of Dehan Ruan in the original version of the paper.

(2) The authors' data should be changed like this erratum

(3) In equation (3.5) the $=$ : should be :=

Keywords and phrases: Markovian switching, prey-predator model, additional food, extinction, unique stationary distribution.

${ }^{1}$ School of Applied Mathematics, Shanxi University of Finance and Economics, Taiyuan, Shanxi 030006, P.R. China.

2 School of Mathematics and Information Sciences, Guangzhou University, Guangzhou, Guangdong 510006, P.R. China.

3 School of Mathematics and Systems Science, Guangdong Polytechnic Normal University, Guangzhou 510665, P.R. China.

4 Guangzhou International Institute of Finance and Guangzhou University, Guangzhou, Guangdong 510405, P.R. China.

* Corresponding author: mathhope@sina.com 
(4) The first equation of Lemma 4.1 (p. 7, -4 line) should be

$$
-\frac{b_{1}(k)\left(a_{1}(k)+\alpha(k) \xi(k)\right)}{\left(a_{1}(k)+\alpha(k) \xi(k)+K\right)^{2}}+d_{1}(k) r(k)-\sum_{j \in \mathcal{M}} \gamma_{k j} d_{1}(j)=0, k=1,2, \cdots, N .
$$

(5) In page 11, line -2 should be

where $F=\frac{M \lambda\left(\hat{a}_{1}+\hat{\alpha} \hat{\xi}\right)}{4 M\left(\check{d}_{1} \check{c}_{1}+d_{4} \ddot{b}_{1}+d_{3} \breve{b}_{1}\right)+\check{b}_{1}}, G_{1}=\left(\frac{(1-v) \hat{\sigma}_{12}\left(\hat{a}_{1}+\hat{\alpha} \hat{\xi}\right)}{4 M\left(\check{d}_{1} \check{c}_{1}+d_{4} \dot{b}_{1}+d_{3} \dot{b}_{1}\right)+\check{b}_{1}}\right)^{\frac{1}{v}}, G_{2}=\left(\frac{(1-v) \hat{\sigma}_{22}\left(\hat{a}_{1}+\hat{\alpha} \hat{\xi}\right)}{4 M\left(\check{d}_{1} \check{c}_{1}+d_{4} \dot{b}_{1}+d_{3} \dot{b}_{1}\right)+\check{b}_{1}}\right)^{\frac{1}{v}}$. 